\title{
Avaliação de Soldagem de Aço Estrutural através do Ruído Magnético de Barkhausen
}

\author{
(Evaluation Of Carbon Steel Welded Plates With Magnetic Barkhausen Noise)
}

\author{
Claudia P. Serna-Giraldo ${ }^{1}$, Linilson R. Padovese ${ }^{2}$ \\ ${ }^{1}$ Universidad de Antioquia, Departamento de Ingeniería Metalúrgica y de Materiales, Medellín, Antioquia, \\ Colombia, claserna@udea.edu.co \\ ${ }^{2}$ Universidade de São Paulo, Departamento de Engenharia Mecânica, São Paulo, SP, Brasi, lrpadove@usp.br
}

\begin{abstract}
Resumo
Este trabalho apresenta resultados de avaliação de juntas soldadas de aço estrutural ASTM A36 por meio do Ruído Magnético de Barkhausen (RMB). A soldagem foi feita em chapas de $6 \mathrm{~mm}$ de espessura com preparação de chanfro em $V$, com um e dois passes. Foram feitas a caracterização de microestructuras e microdurezas através da junta soldada, e na superfície foram feitas medições de RMB. Os sinais de RMB foram analisados com o valor máximo, uma média de valores acima de $80 \%$ do pico máximo, o rms e o rms do envelope, dos quais foram obtidas curvas da variação com respeito ao centro do cordão e também foram obtidos mapas superficiais. $\mathrm{Na}$ caracterização microestructural obteve-se que as amostras de um e dois passes apresentaram tamanhos da zona afetada pelo calor (ZAC) diferente. As microdurezas mostraram que no limite de fusão tem-se a maior dureza enquanto que no fim da ZAC tem-se a menor dureza. O RMB mostrou que no limite de fusão tem o menor valor, enquanto que no fim da ZAC tem o maior valor. Este estúdio mostrou que as mudanças na microestrutura influenciam em todos os parâmetros analisados, sendo que a junta soldada ficou melhor representada pelo valor rms e rms do envelope do $R M B$.
\end{abstract}

Palavras-chave: Ruído Magnético de Barkhausen (RMB). Soldagem de aços Carbono. Ensaios Não Destrutivos Magnéticos.

Abstract: This paper shows results for the evaluation of ASTM A36 carbon steel welded joints by Magnetic Barkhausen Noise (MBN). $V$-groove shape welded samples were made in a $6 \mathrm{~mm}$ thickness plates, with one-pass and two-pass. Microstructural and microhardness characterization were made on a transversal section of welds. In the surface was made measurement of MBN. The signal of MBN were analyzed with the maximum value, threshold of $80 \%$ above of maximum, the root mean square (rms), the root mean square of the profile, and these parameters were plotted in function of the distance to center bead. Surface maps were obtained, too. The microstructural characterization identified different heat affected zone (HAZ) sizes for one-pass and two-pass. In addition, changes on microhardness showed which higger hardness was happened in the fusion boundary whereas lower microhardness happened in the HAZ end. With $M B N$ monitoring were verified that the lower value happened in the fusion boundary, whereas the MBN higher value happened in the HAZ end. The study showed that changes in the microstructure influence all analyzed parameters. The welded joint are best represented by the root mean square ( $r m s)$, the root mean square of the profile.

Key-words: Magnetic Barkhausen Noise (MBN). Carbon Steel Welding. Magnetic Non-destructive Testing.

\section{Introdução}

Os materiais ferromagnéticos, ao serem submetidos a campos magnéticos variáveis, experimentam um aumento na indução em forma descontínua, como resposta aos processos de movimentação das paredes de domínios magnéticos. Estes aumentos abruptos de indução são conhecidos como Ruído Magnético de Barkhausen (RMB). Os sinais são detectados como pulsos de tensão quando uma bobina é colocada perto da superfície da amostra enquanto acontece o movimento das paredes de domínio. O RMB é sensível às características

(Recebido em 17/05/2010; Texto final em 23/09/2010). do material como a microestrutura e às tensões mecânicas aplicadas, fazendo com que seu uso seja promissor como ensaio não destrutivo. Este trabalho apresenta a utilização do RMB na caracterização de juntas soldadas de aço estrutural ASTM A36. Os resultados mostram a relação de alguns parâmetros calculados do sinal de RMB com a variação microestrutural na junta soldada.

\subsection{Metalurgia de Soldagem de Aços Carbono.}

Numa junta soldada de aço carbono, a microestrutura varia ponto a ponto desde o cordão de solda até o metal base como conseqüência dos ciclos térmicos experimentados, em função dos parâmetros empregados na soldagem [1]. Podemse identificar assim a zona de fusão, a zona afetada pelo calor (ZAC) e o metal base não afetado. Na zona de fusão acontecem fenômenos de solidificação e resfriamento desde a temperatura 
de fusão, e assim as microestructuras são típicas de solidificação. Na zona adjacente ao cordão de solda, a temperatura não atinge a de fusão e, assim, no metal ocorrem transformações de fase em estado sólido desde altas temperaturas, devido aos ciclos de aquecimento e resfriamento. A microestrutura está determinada unicamente pelos ciclos térmicos a que ela é submetida, sendo que a temperatura máxima atingida em cada ponto varia conforme aumenta a distância ao centro do cordão de solda. Em aços carbono, a ZAC divide-se em três zonas: supercrítica, intercrítica e subcrítica. Na zona supercrítica, tem-se a região de crescimento de grão, que corresponde àquela na qual a temperatura máxima do ciclo térmico excede a temperatura de crescimento de grão. Conforme se afasta da linha de centro do cordão de solda, tanto as temperaturas máximas quanto as velocidades de resfriamento diminuem, tendo assim uma gradiente de microestruturas na região de grão refinado que são similares ao do aço normalizado do metal base. Abaixo da temperatura de crescimento de grão, o ciclo térmico geralmente produz um tamanho de grão menor do que o metal base. Na região intercrítica que é relativamente estreita, acontece uma transformação parcial e a microestrutura consiste de colônias de perlita imersas numa matriz ferritica não transformada. Na região subcrítica geralmente não acontece mudança microestrutural apreciável, exceto que pode ocorrer uma pequena região de esferoidização, mas esta é difícil de detectar. [2]

\subsection{O Ruído Magnético de Barkhausen (RMB)}

Nos materiais ferromagnéticos, existem grandes regiões nas quais os momentos magnéticos atômicos estão alinhados segundo a direção de magnetização espontânea, e se conhecem como domínios magnéticos. A direção de alinhamento dos momentos magnéticos, varia de domínio a domínio, segundo a direção do eixo de fácil magnetização. A separação entre os domínios magnéticos é uma camada de transição conhecida como paredes de domínio, ou paredes de Bloch. Em materiais cúbicos, estas são de $180^{\circ}$ ou de $90^{\circ}$.
Ao aplicar um campo magnético variável a um material ferromagnético, tem-se observado que próximo do campo coercitivo $\left(\mathrm{H}_{\mathrm{c}}\right)$, o aumento da indução, em decorrência do aumento de $\mathrm{H}$, não ocorre de maneira contínua, mas sim a maneira de saltos abruptos conhecidos como saltos de Barkhausen, como se ilustra na Figura 1a. A ocorrência destes saltos abruptos tem sido explicada pela interação da movimentação das paredes de domínio e das barreiras a essa movimentação nos pontos de ancoragem (constituídos pelas imperfeições do material como discordâncias, contornos de grão, precipitados) [3]. As emissões de Barkhausen geram o sinal de RMB como mostrado na Figura $1 \mathrm{~b}$, e podem ser detectadas como pulsos de tensão elétrica, se uma bobina for colocada perto da superfície da amostra enquanto acontece o movimento das paredes dos domínios.

O RMB depende dos parâmetros empregados para a magnetização, como a frequiência [3-56], e a intensidade de magnetização $[7,8]$ mas também depende das características do material como o tamanho de grão [9-101112], o teor de carbono $[13,14]$ e o estado de tensões $[15,16]$.

Uma das potencialidades do RMB é a sua utilização no monitoramento de peças soldadas para identificar variações estruturais, efeito de tratamentos térmicos ou presença de defeitos. Alguns dos trabalhos de RMB em soldagem tem sido feitos em microestruturas simuladas através de tratamentos térmicos [17-1819], e outros tem sido realizados em soldagens reais para avaliar o efeito de tratamento térmico pós-soldagem [20] ou o desempenho da junta soldada à fadiga [21-2223]. Neste trabalho avalia-se a variação microestructural de juntas soldadas de aço carbono utilizando o RMB.

\section{Materiais e Métodos}

O material de estudo é uma chapa de aço estrutural A36 comercial de $6 \mathrm{~mm}$ de espessura. A composição química foi obtida através de análise por espectrometria de emissão óptica e apresenta-se na Tabela 1.

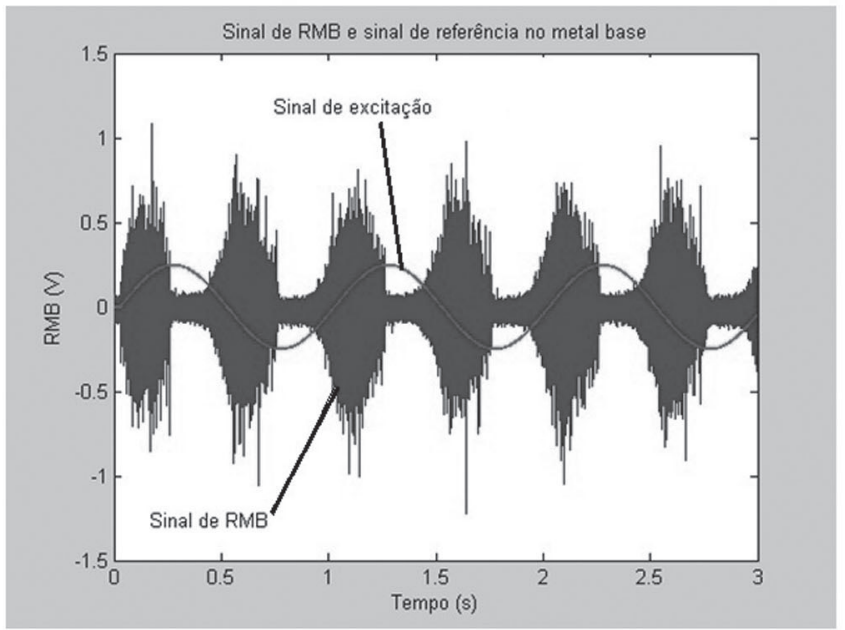

b)

a)

Figura 1. a) Curva de magnetização de um ferromagnético [4], b) sinal de Ruído magnético de Barkhausen. 
Tabela 1. Composição química do aço estrutural A

\begin{tabular}{ccccccccc}
\hline $\mathrm{Fe}$ & $\mathrm{C}$ & $\mathrm{Si}$ & $\mathrm{Mn}$ & $\mathrm{S}$ & $\mathrm{P}$ & $\mathrm{Ni}$ & $\mathrm{Cr}$ & Mo \\
\hline 98,323 & 0,111 & 0,308 & 0,989 & 0,032 & 0,011 & 0,029 & $0,098 \mathrm{R}$ & 0,050 \\
\hline
\end{tabular}

Tabela 2. Parâmetros de soldagem empregados para preparação das amostras

\begin{tabular}{ccccccc}
\hline $\begin{array}{c}\text { Quantidade } \\
\text { passes }\end{array}$ & Passe No & Tensão (V) & Corrente (A) & $\begin{array}{c}\text { Velocidade } \\
(\mathrm{mm} / \mathrm{s})\end{array}$ & $\begin{array}{c}\text { Aporte térmico }(\mathrm{J} / \\
\mathrm{mm})\end{array}$ & $\begin{array}{c}\text { Técnica } \\
\text { cordão }\end{array}$ \\
\hline 1 & 1 & $27-32$ & $160-165$ & 2,3 & $1502-1837$ & Oscilado \\
2 & 1 & $20-22$ & $108-115$ & 3,07 & $619-659$ & Reto \\
& 2 & $20-22$ & $108-115$ & 2,10 & $822-963$ & Oscilado \\
\hline
\end{tabular}

As amostras soldadas foram preparadas com chapas de 100 $\mathrm{mm}$ de largura por $100 \mathrm{~mm}$ de comprimento, com preparação de chanfro em V. A soldagem foi feita com o processo de arco metálico com eletrodo revestido (SMAW). O eletrodo utilizado foi o E7018 de 3,2 mm de diâmetro. Prepararam-se amostras de um passe e de dois passes com o propósito de obter tamanhos de ZAC diferentes. A Tabela 2 mostra os parâmetros que foram utilizados para realizar as soldas.

A fim de se obter uma superfície plana, o excesso de solda foi retirado mediante processo de retifica, com um passo de $0,05 \mathrm{~mm}$ em cada passada e 0,03 na última passada. Para a caracterização microestrutural, uma seção transversal das juntas soldadas foram cortadas e preparadas metalograficamente com lixamento até lixa 1000 e polimento com pasta de diamante até 1 mícron, depois foram atacadas com nital $2 \%$ e observadas no microscópio ótico. As dimensões da zona de fusão e da zona afetada pelo calor foram medidas na seção transversal a um milímetro abaixo da superfície. Nesta mesma linha, foram feitas medidas de microdureza a partir do centro do cordão até uma distância de $15 \mathrm{~mm}$, separadas a cada $1 \mathrm{~mm}$. Usou-se indentador Vickers com carga de $100 \mathrm{~g}$.

Os sinais de Ruído Magnético de Barkhausen na superfície das chapas foram obtidos através da cadeia de medição desenvolvida no Laboratório de Dinâmica e InstrumentaçãoLADIN da Universidade de São Paulo. Este é consistente de três sistemas básicos: um conjunto excitador-sensor (que constitui a sonda), o condicionador de sinal e o sistema de aquisição e tratamento de dados. Na Figura 2 mostra-se um esquema do sistema de medição.

Por meio de uma fonte bipolar Kepco BOP 20-20 D, gerase uma corrente variável que passa através da bobina do yoke, gerando um campo magnético variável no yoke magnético de até $12500 \mathrm{~A} / \mathrm{m}$, com uma frequência de $1 \mathrm{~Hz}$. A corrente da fonte Kepco é controlada por um sinal de controle de onda senoidal gerado no microcomputador e enviada à fonte pela saída D/A do sistema de aquisição. No condicionador, o sinal é filtrado por um filtro passa banda de quarta ordem (de 1-100 kHz) e amplificado por um amplificador de ganho variável programável até 8000 vezes. Os dados são adquiridos numa placa de aquisição de dados de 12 bits, com uma frequiência de amostragem de 200 $\mathrm{kHz}$. Em cada ponto foram adquiridos 5 sinais de RMB.

Foram calculados o valor de pico máximo, a média acima de $80 \%$ do valor máximo, o rms (root mean square) e o rms do envelope e levantados mapas superficiais que se relacionaram com o estado microestrutural.

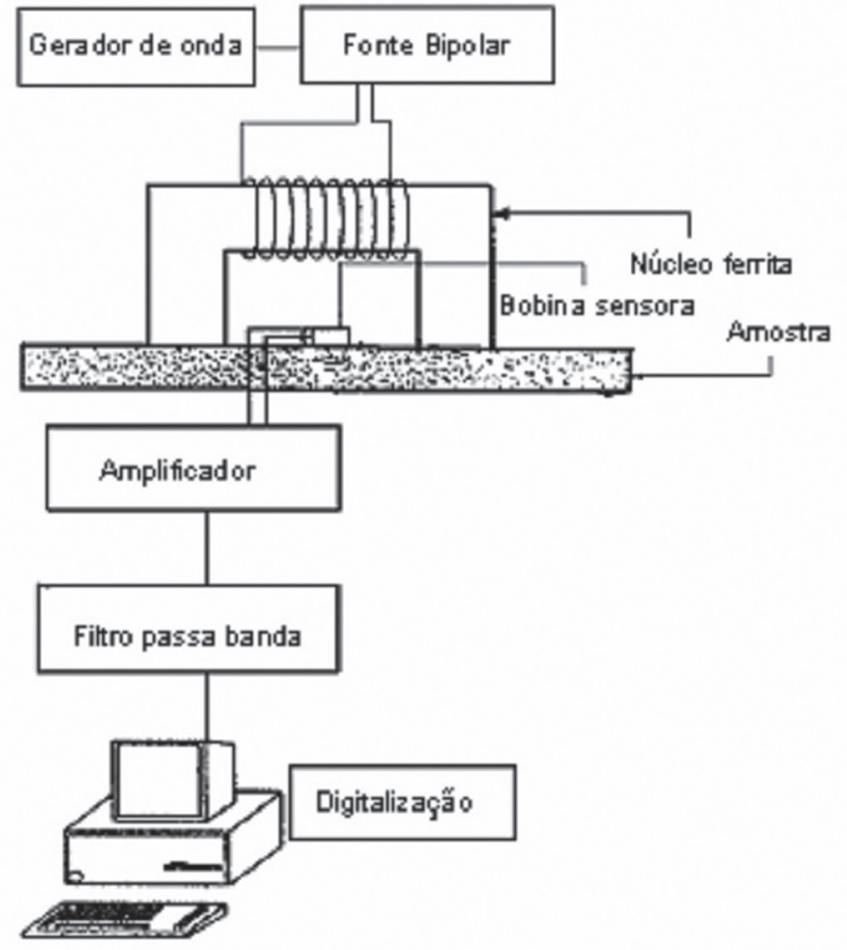

Figura 2. Esquema do sistema de medição do RMB

\section{Resultados e Discussão}

A junta soldada consiste de uma zona de fusão, uma zona afetada pelo calor e o metal base não afetado. A Figura 3 mostra as macrografias da seção transversal das amostras de um passe e de dois passes com suas regiões. A dimensão das zonas, a partir do centro do cordão em cada uma das amostras se apresenta na Tabela 3. Na Figura 4 apresentam-se as micrografias de cada uma das regiões da junta soldada da amostra de um passe, que são análogas às da amostra de dois passes.

Tabela 3. Dimensões das regiões da junta soldada a partir do centro do cordão

\begin{tabular}{|c|c|c|}
\hline Amostra & $\begin{array}{c}\text { Largura zona } \\
\text { de fusão }(\mathrm{mm})\end{array}$ & $\begin{array}{c}\text { Largura da } \\
\text { ZAC }(\mathrm{mm})\end{array}$ \\
\hline 1 passe & 5 & 5 \\
\hline 2 passes & 4 & 2 \\
\hline
\end{tabular}




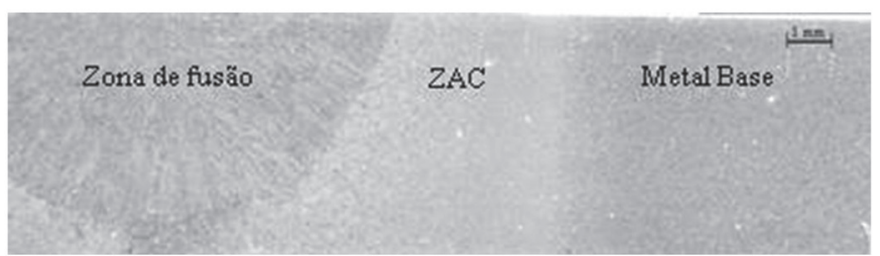

a)

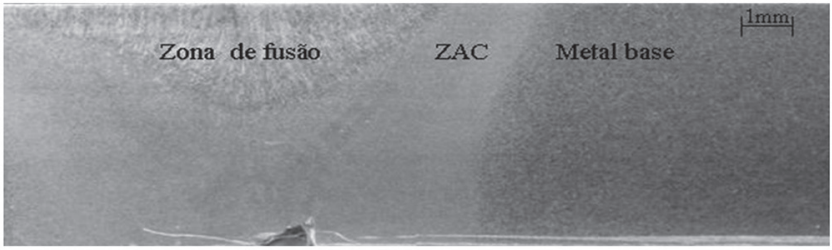

b)

Figura 3. Macrografias das juntas soldadas, a) um passe, b) dois passes.

Na zona de fusão (Figura 4a) a microestrutura é típica de uma estrutura de solidificação. Percebe-se a orientação dos grãos que cresceram epitaxialmente em direção ao centro da solda. A microestrutura consiste de ferrita primária no contorno de grão e também poligonal, com placas de Widmanstätten nucleadas no contorno de grão. Ao interior do grão tem-se ferrita acicular com pouca perlita no espaçado entre as agulhas. A região de crescimento de grão é estreita (Figura 4b), e sua microestrutura consiste de ferrita primária no contorno de grão a partir do qual crescem placas de ferrita Widmanstätten. No interior do grão tem-se uma estrutura de ferrita acicular com perlita. Conforme se afasta da linha de fusão, a microestrutura muda tanto no tamanho do grão, o qual diminui, como também na morfologia da ferrita que vai se tornando poligonal. Isto é devido às menores temperaturas máximas atingidas nessa região que evitam o crescimento do grão austenítico, e às menores velocidades de resfriamento que evitam a formação de microestructuras de forma acicular. Nessa região de grão intermediário (Figura 4c) a microestrutura consiste de ferrita e pouca perlita. Mais afastado da linha de fusão, tem-se a região de grão refinado (Figura 4d), na qual a temperatura máxima atingiu a de austenização, mas foi menor. Assim, o grão austenítico não conseguiu crescer e a velocidade de resfriamento foi também mais baixa, a microestrutura é composta basicamente de ferrita poligonal e perlita. Na zona intercrítica (Figura 4e), a perlita transformou a austenita durante o aquecimento, e a ferrita mais perlita durante o resfriamento, a microestrutura resultante são colônias pequenas de perlita imersas numa matriz ferrítica. Contígua a esta última zona, tem-se a região do metal base não afetado (Figura 4f), na qual as temperaturas máximas atingidas estiveram abaixo da linha de austenização A1, e por tanto não houve transformação de fase. Provavelmente aconteceu uma esferoidização da perlita, mas esta não se resolve pela microscopia óptica. A microestrutura consiste de ferrita e perlita.

$\mathrm{Na}$ Figura 5 mostram-se os perfis de microdureza das amostras soldadas. Percebe-se como na região correspondente à zona de fusão, a microdureza é bastante heterogênea, devido às heterogeneidades da microestrutura. No limite de fusão se tem o valor maior de dureza devido à microestrutura acicular que

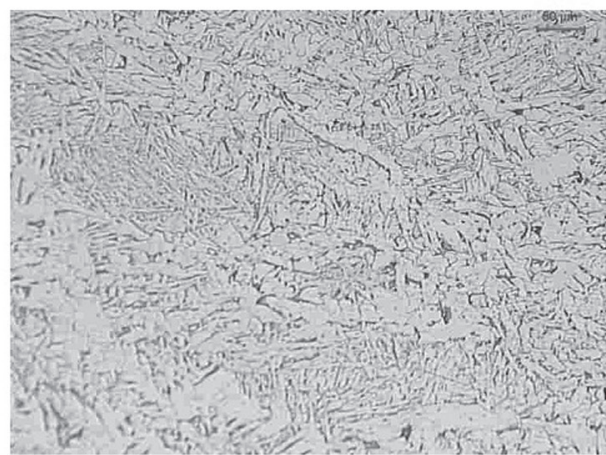

a)

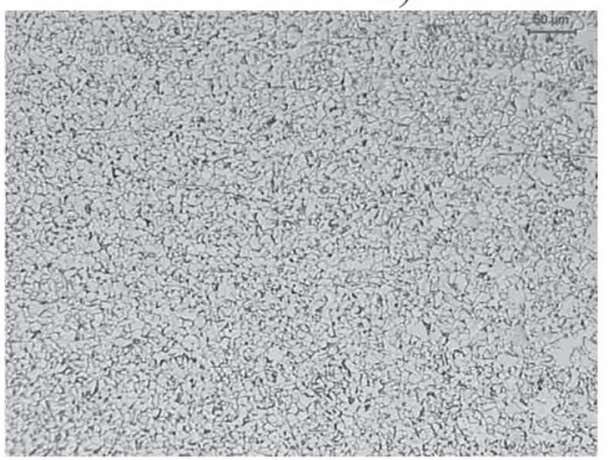

d)

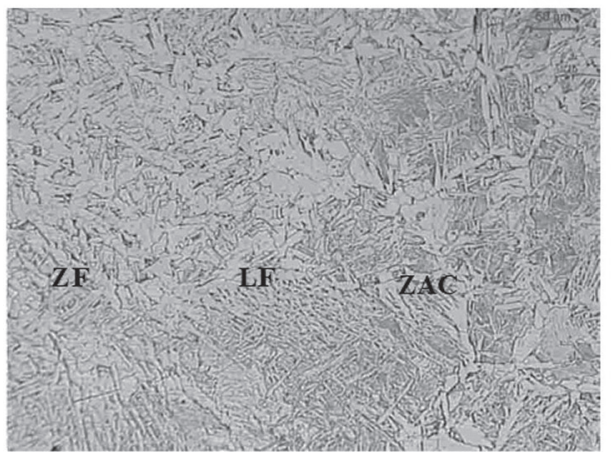

b)

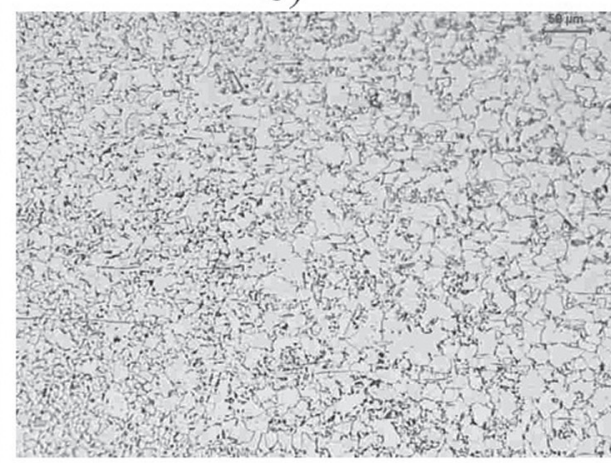

e)

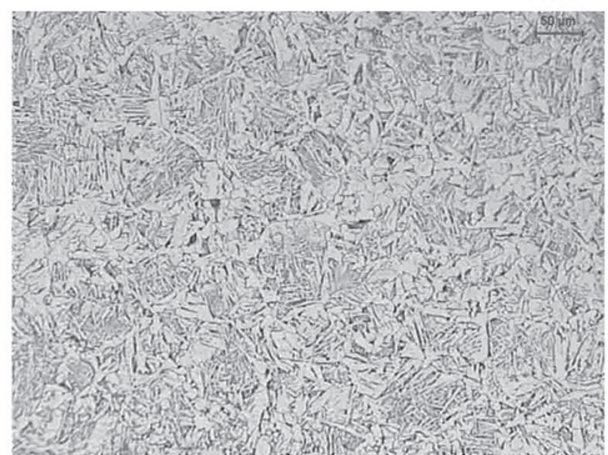

c)

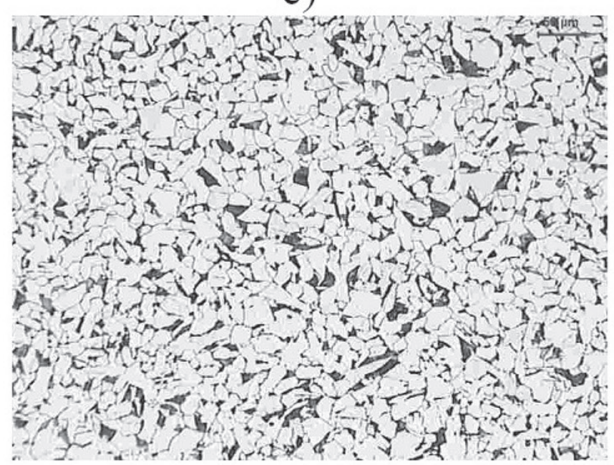

f)

Figura 4. Microestructuras das regiões da junta soldada. a) zona de fusão, b)zona de crescimento de grão, c) zona de grão intermediário, d) zona de refinamento de grão, e) zona de transformação parcial, f) zona de metal base não afetado. 


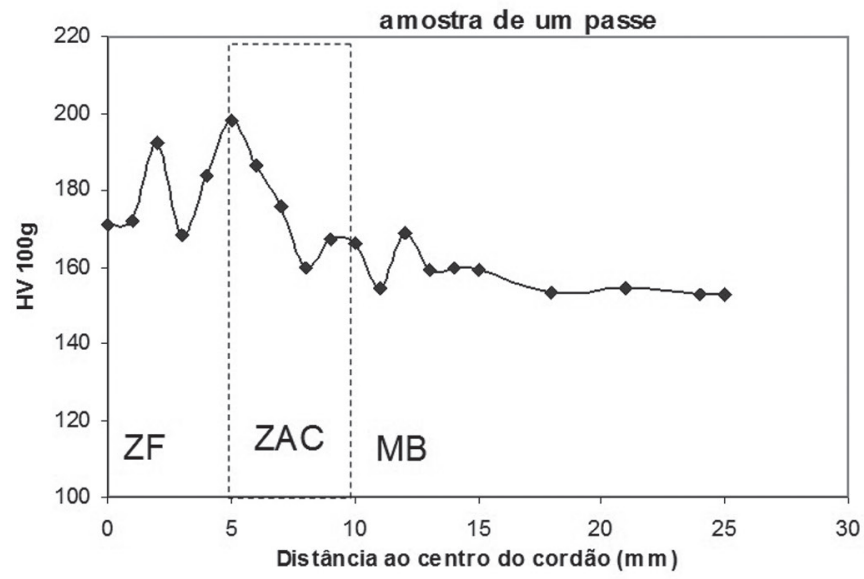

a)

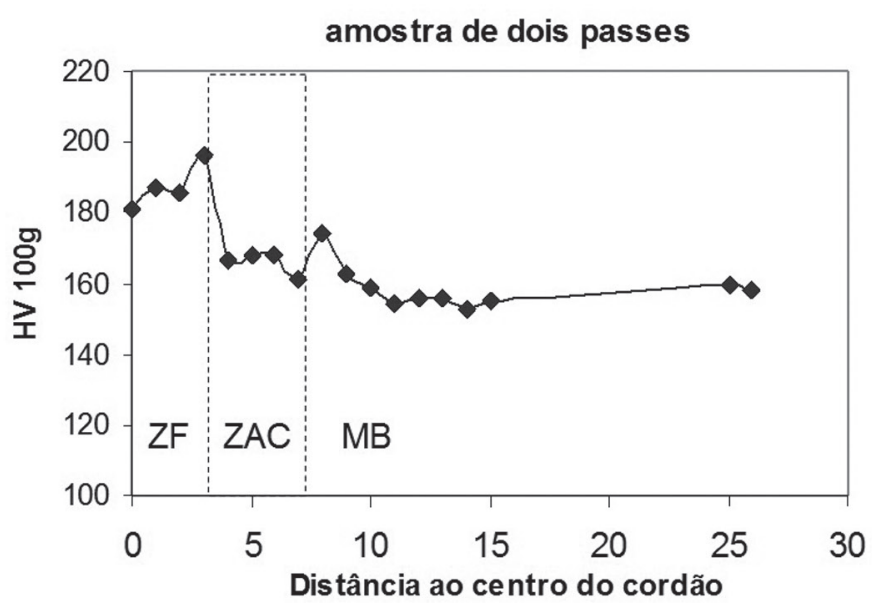

b)

Figura 5. Perfis de microdureza das amostras soldadas, a) um passe, b) dois passes

apresenta. Depois a dureza sofre uma queda até o fim da ZAC que apresenta o valor mínimo. Depois aumenta um pouco e cai novamente até que se estabiliza no metal base.

$\mathrm{Na}$ Figura 6 mostram-se os valores máximo, média de valores acima de $80 \%$ do valor máximo, o rms e o rms do envelope da amostra de um passe, em função da distância ao centro do cordão. Cada curva representa uma linha medida na superfície da chapa, transversal ao cordão de solda desde o centro do cordão até o metal base na distância de $35 \mathrm{~mm}$.

Na Figura 7 mostram-se estes mesmos parâmetros para a amostra de dois passes.

Observa-se das Figuras 6 e 7 que não há uma grande diferença entre as curvas dos diferentes parâmetros. O comportamento é o mesmo, e a diferença é apenas nas amplitudes. As curvas dos valores máximos e $80 \%$ acima do valor máximo são muito flutuantes, em quanto que as curvas do rms e do rms do envelope são mais suaves, facilitando a localização das diferentes regiões.

Nas amostras de um passe, observa-se que o valor do sinal de Ruído Magnético de Barkhausen diminui a partir do centro do cordão de solda, até um vale entre 5-7 mm no qual se tem o valor mínimo, este corresponde à linha de fusão (LF). A partir deste ponto, percebe-se um aumento até 10-12 $\mathrm{mm}$, que corresponde com o fim da ZAC. Nas amostras de dois passes observa-se a presença de um vale na distância de $4-5 \mathrm{~mm}$, a partir da qual acontece um incremento no sinal, até a distância de $7 \mathrm{~mm}$ na qual se apresenta um máximo relativo, depois há uma queda, e novamente o RMB aumenta, até um novo valor máximo $26 \%$ maior do que o primeiro, tem uma nova queda e se estabiliza. Percebe-se também que os valores das amostras de dois passes são 40-50\% menores que os valores das amostras de um passe

Os comportamentos dos parâmetros de RMB se relacionam com as variações de microdureza. Assim, no limite de fusão, no qual se tem uma microestrutura acicular com maior densidade de discordâncias, teve-se o maior valor de dureza, e obteve-se o menor valor do parâmetro do sinal de RMB. Isto concorda com o reportado que quando maior é o valor de dureza, o RMB é menor[6]. As discordâncias agem como barreiras à movimentação das paredes de domínio, por tanto a emissão de RMB é baixa. Na medida em que se afasta da linha de fusão, a velocidade de resfriamento diminui favorecendo uma mudança da morfologia acicular da ferrita a qual se torna poligonal, com presença de colônias de perlita mais grossa. Também se observa uma diminuição no tamanho de grão da ferrita e da perlita. Estas microestruturas apresentam menor dureza. Na medida em que o grão vai se refinando, aumenta o número de paredes de domínio se movimentando, além de que a não existência da estructura acicular faz com que a quantidade de discordâncias diminua, diminuindo a quantidade de barreiras à movimentação dos domínios, facilitando-se assim a movimentação das paredes de domínio. Esta interação produz uma maior emissão de RMB.

Mapas superficiais correspondentes ao valor rms das amostras de um passe e de dois passes são mostrados na Figura 8. Nessas superfícies percebe-se claramente uma faixa até a distância de $10 \mathrm{~mm}$ (na amostra de um passe) e de $7 \mathrm{~mm}$ ( na amostra de dois passes). Estas faixas representam a zona soldada, ou seja, o cordão de solda e a ZAC. A partir desta distância tem-se uma região de cores mais homogêneas que representam o metal base. Estes mapas superficiais permitem localizar a região correspondente à solda, tornando-se muito úteis como uma ferramenta não destrutiva para monitorar as variações microestruturais na junta soldada. 


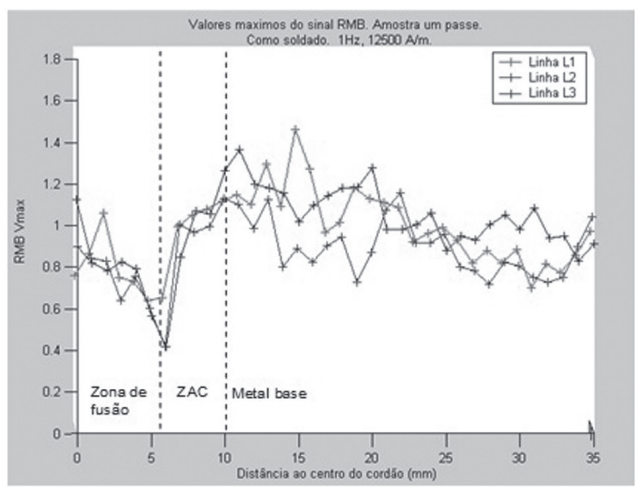

a)

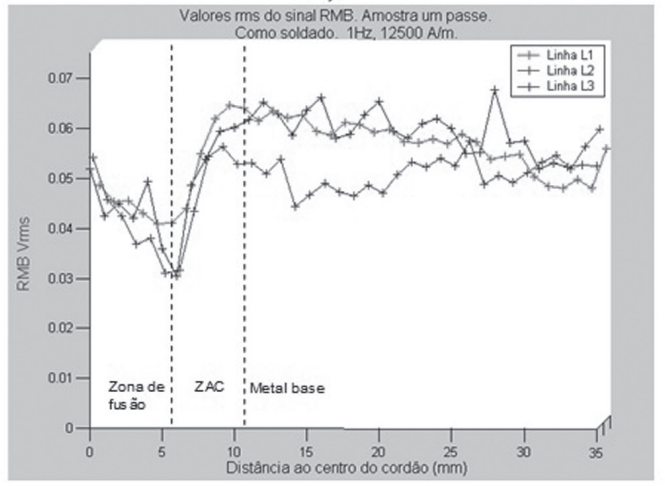

c)

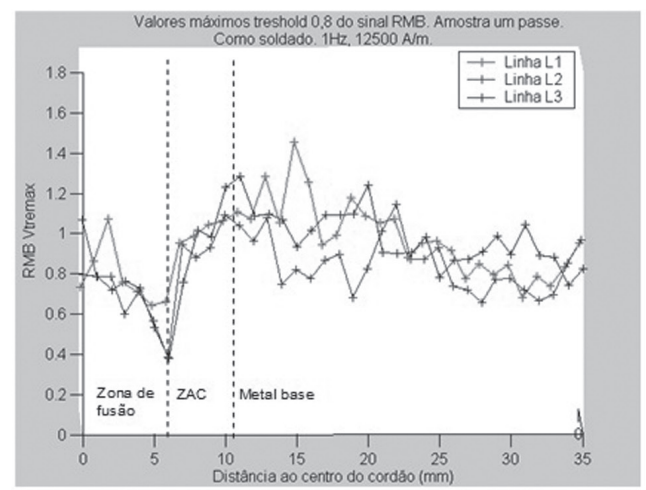

b)

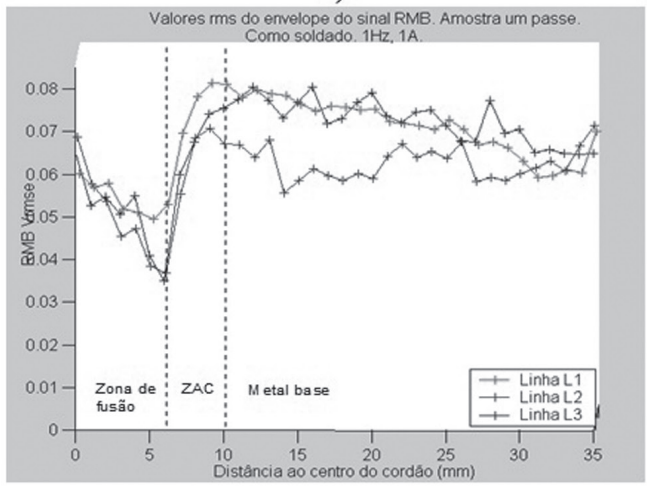

d)

Figura 6. Parâmetros de RMB da amostra de um passe a)valor máximo, b) média de valores acima de $80 \%$ do máximo, c) rms, d) rms do envelope.

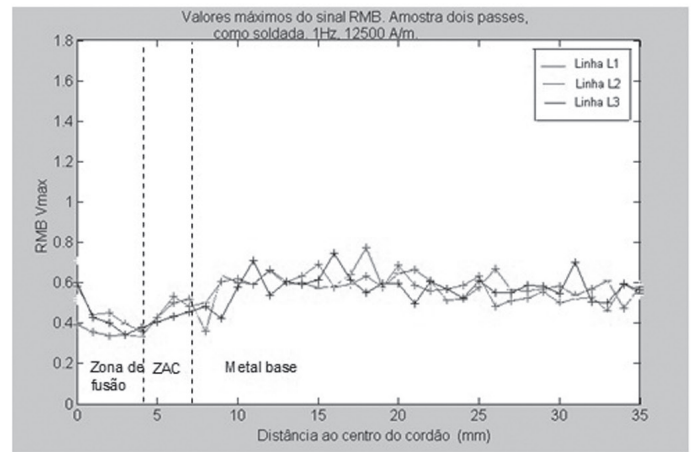

a)

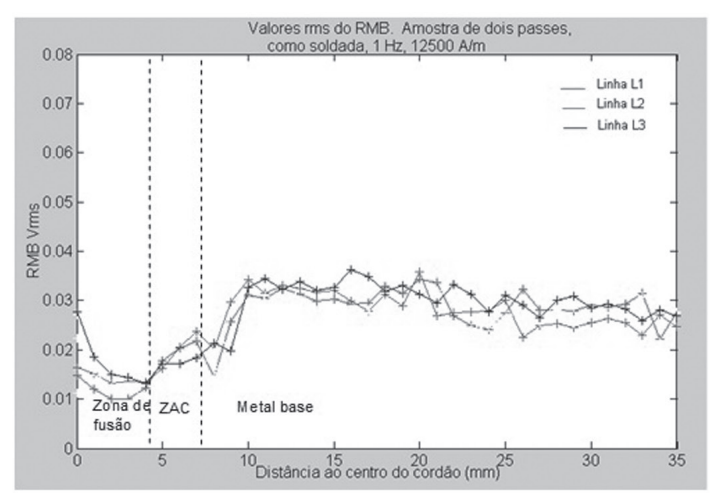

c)

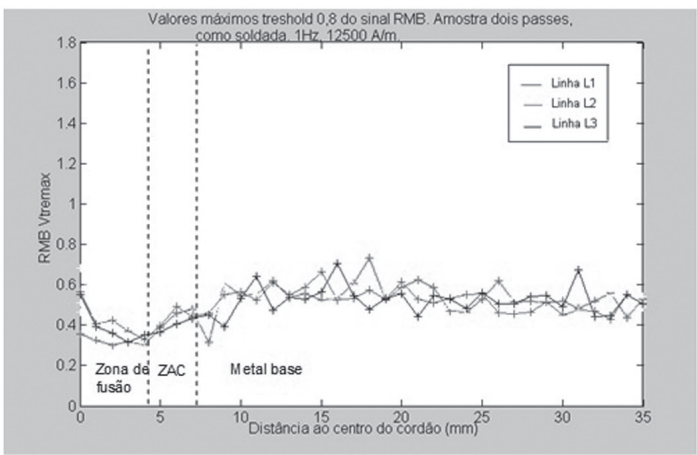

b)

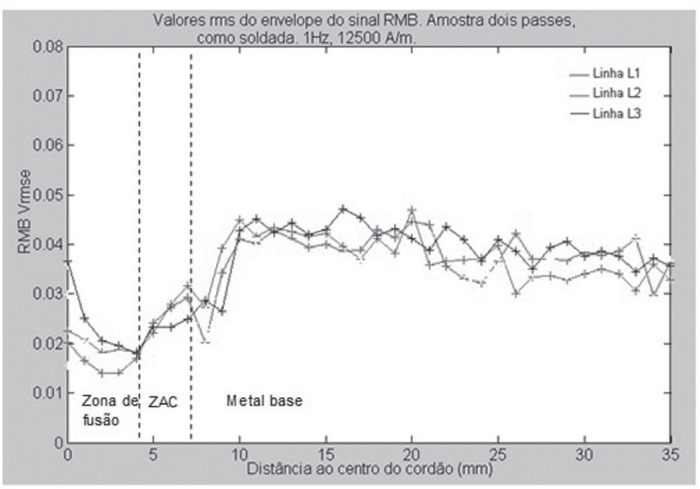

d)

Figura 7. Parâmetros de RMB da amostra de dois passes a)valor máximo, b) média de valores acima de $80 \%$ do máximo, c) rms, d) rms do envelope 


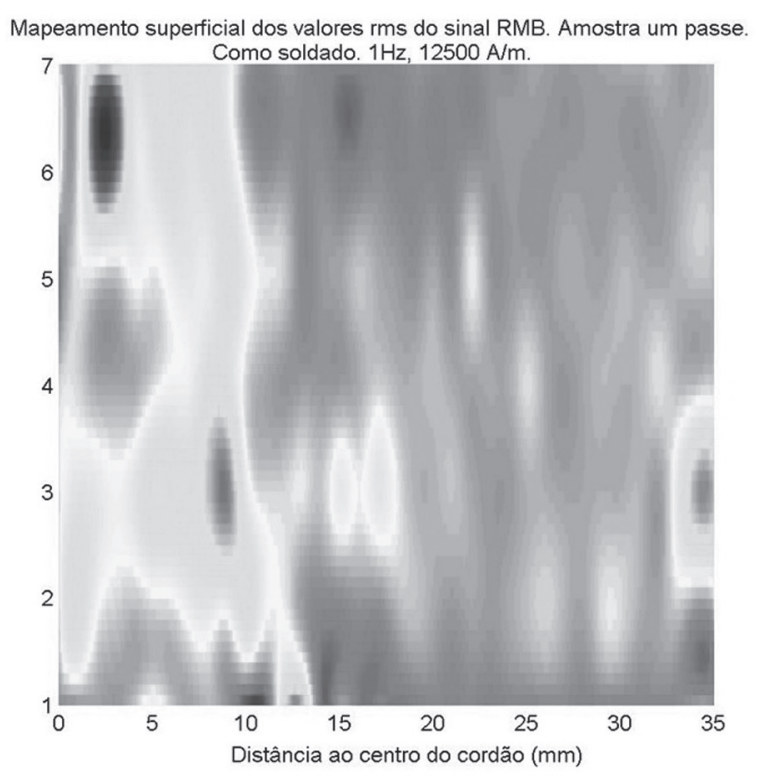

a)

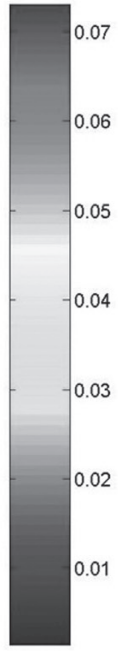

Figura 8. Mapas superficiais do rms do RMB a) amostra de um passe, b) amostra de dois passes.

\section{Conclusões}

O trabalho realizado permitiu identificar as diferentes regiões em juntas soldadas de aço carbono de um e dois passes, mostrando a sua variação microestrutural e de durezas desde o centro do cordão até o metal base. Essa variação microestrutural foi relacionada com sinais de RMB, que também apresentaram variação através da junta soldada. Assim, no limite de fusão onde se teve o maior valor de dureza, o parâmetro de RMB foi menor. No fim da ZAC na qual se mediram os menores valores de dureza, o parâmetro de RMB foi o maior. Dos parâmetros analisados constatou-se que todos têm informação da variação microestrutural, sendo que o valor rms e o rms do envelope são os que representam essa variação de forma mais suave. Com os parâmetros de RMB foi possível levantar mapas superficiais que diferenciam a região correspondente à solda, da região correspondente ao metal base. Estes resultados mostram a potencialidade de utilizar o RMB como ferramenta não destrutiva na avaliação microestrutural de juntas soldadas.

\section{Agradecimentos}

Os autores agradecem a CAPES e à Universidade de Antioquia pelo apoio financeiro.

\section{Referências Bibliográficas}

[1] GRANJON, H. Fundamentals of Welding Metallurgy. Abigton Publishing. Cambridge. 1991. pp18-40.

[2] LANCASTER, J.F. Metallurgy of welding. Chapman \& Hall. London, 1994. p175-198

[3] JILES, D.C. Dynamics of domain magnetizatin and the Barkhausen effect. Czechoslovak Journal of Physics. V 50, n 8, 2000. pp 893-988.

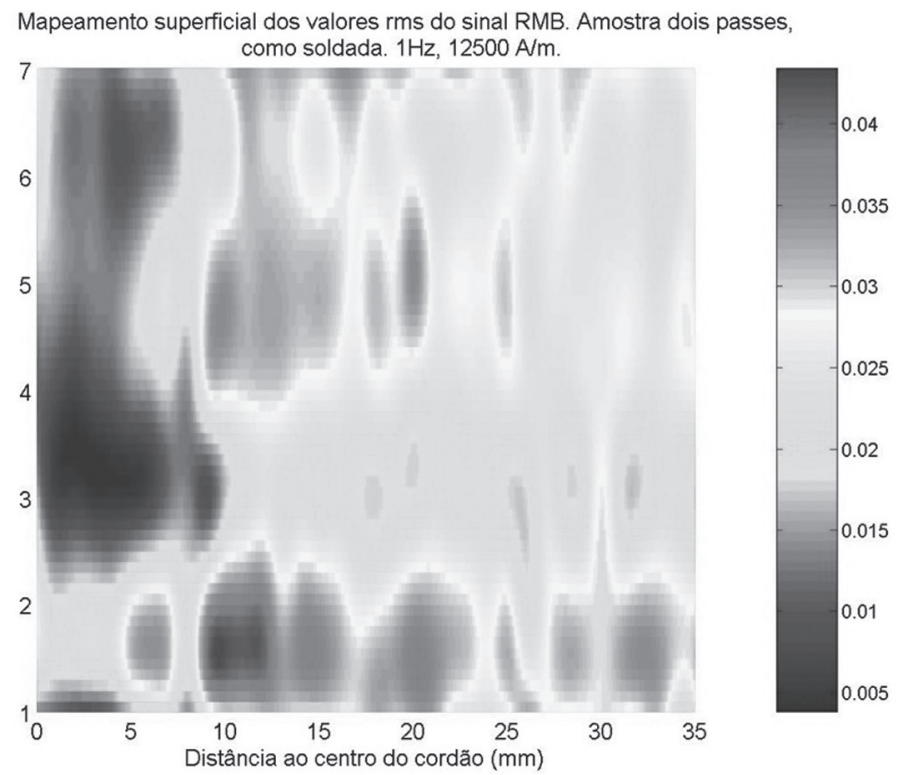

b)

[4] JILES, DAVID. Introduction to magnetism and magnetic materials. Chapman and Hall. New York.1994.

[5] ALTPETER, I. Nondestructive evaluation of cementite content in steel and white cast iron using inductive Barkhausen noise. Journal of Nondestructive Evaluation. V15, n2. 1996. pp 45-60

[6] MOORTHY, V.; SHAW, B.A.; EVANS, J.T. Evaluation of tempering induced changes in the hardness profile of casecarburised EN steel using magnetic Barkhausen noise analysis. NDT\&E international. V 36, 2003, pp 43-49.

[7] DHAR, A. ATHERTON,D.L. Influence of Magnetizing Parameters on the Magnetic Barkhausen Noise. IEEE Transactions on Magnetics. V28, N 6.1992. pp 3363-3366.

[8] PADOVESE, L.R., CAPÓ, J. ANGLADA-RIVERA, J. Medição da tensão mecânica em aço carbono através da emissão magnética de Barkhausen. . In: XIX Congresso Nacional de Ensaios Não Destrutivos. São Paulo, Brasil, agosto 2000.

[9] RANJAN, R.; JILES, D.; RASTOGI, P.K. Magnetic properties of decarburized steels: an investigation of the effects of grain size and carbon content. IEEE transactions on magnetics. Vol 23. n 3, 1987. pp 1869-76.

[10]GATELIER-ROTHEA, C.;FLEISCHMANN, P.; CHICOIS,J.; FOUGERES. Role of microestructural states on the level of Barkhausen noise in pure iron and low carbon iron binay alloys. Nondestructive Test Evaluation. V 8-9. 1992, pp 591-602.

[11] CAPÓ-SANCHEZ, J, PADOVESE, L.R., SERNAGIRALDO, C.P. Utilización del efecto Barkhausen en el análisis estructural de muestras de acero al carbono. In: II Congresso Nacional de Engenharia Mecânica. João Pessoa, Brasil, Agosto 2002.

[12] CAPÓ-SANCHEZ, J, PADOVESE, L.R., SERNAGIRALDO, C.P. Medição do ruído magnético Barkhausen em aços carbono. In: 57 congresso anual da ABM Internacional. 
São Paulo, Brasil, julio de 2002.

[13] KAMEDA, J.; RANJAN,R. Nondestructive evaluation of steels using acoustic and magnetic Barkhausen signals-I. Effect of carbide precipitation and hardness. Acta Metallurgical. V35, n 7. 1987. pp 1515-26.

[14] CAPÓ-SANCHEZ J, et al. Dependence of the Barkhausen Magnetic Emission with carbon content in commercial steel. Journal of Materials Science. V39, 2004. pp 1367-1370.

[15] SIPAHI, L.B. Overview of applications of micromagnetic Barkhausen emissions as noninvasive material characterization technique. Journal Applied Physics. V70, n 10. May 1994. pp 6978-80

[16] ANGLADA-RIVERA, J. PADOVESE, L.R., CAPÓSANCHEZ, J. Magnetic Barkhausen noise and hysteresis loop in commercial carbon steel: influence of applied tensile stress and grain size. Journal of Magnetism and Magnetic Materials. V 231, 2001. pp 299-306.

[17] MOORTY,V.,et al. Evaluation of microstrutures in 2,25Cr$1 \mathrm{Mo}$ and 9Cr-1Mosteel weldments using magnetic Barkhausen noise. Materials Science and Engineering. A231, 1997. Pp 98104

[18] JOO-HAG KIM, et al. Effect of microestructurre on magnetic Barkhausen noise level in the weld HAZ of an RPV steel. Journal of Magnetism and Magnetic Materials. V 196197, 1999. pp351-353.

[19] DUCK GUN-PARK, CHEOL GI KIM, JUN-HWA HONG. Microestructural dependence of Barkhausen noise and magnetic relaxation in the weld HAZ of an RPV steel. Journal of Magnetism and Magnetic Materials. V 215-216, 2000. pp765768.

[20] MOORTY, V, et al. Evaluation of post-weld heat treatmente in $2,25 \mathrm{Cr}-1 \mathrm{Mo}$ steel tube to tube sheet welded joints using magnetic Barkhausen noise measurement. Materials Science and Technology. V13, n.7. 1997. Pp614-617.

[21]LACHMANN, C. NITSCHKE-PAGEL, TH, WOHLFAHRT, H. Characterisation of residual stress Relaxation in Fatigue Loaded Welded Joints by X-Ray Diffraction and Barkhausen Noise Method. Materials Science Forum. V 347-349, 2000. pp 374-379

[22] LACHMANN, C. NITSCHKE-PAGEL, TH, WOHLFAHRT, $\mathrm{H}$. Characterization of the fatigue of Cyclically Loaded Welded Joints by Micromagnetic Testing and X-Ray Diffraction. In: Proceedings First Internacional Conference in Barkhausen Noise ICBM 1. Hannover, Germany, 1999.

[23] LACHMANN, C. NITSCHKE-PAGEL, TH, WOHLFAHRT, $\mathrm{H}$. Nondestructive characterization of residual stress relaxation and fatigue processes in cyclically loaded welded joints. In: Proceedings of the 6th international conference on residual stress. Oxford.U.K. 2000, pp1400-1409 